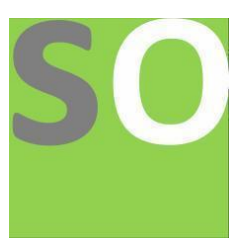

Article title: Growth in Internet Usage During the COVID-19 Pandemic among Older Adults: Findings from Switzerland Authors: Alexander Seifert[1]

Affiliations: Center of Gerontology, University of Zurich[1]

Orcid ids: 0000-0003-3124-4588[1]

Contact e-mail: alexander.seifert@zfg.uzh.ch

License information: This work has been published open access under Creative Commons Attribution License $\mathrm{http}: / / c r e a t i v e c o m m o n s . o r g / l i c e n s e s / b y / 4.0 /$, which permits unrestricted use, distribution, and reproduction in any medium, provided the original work is properly cited. Conditions, terms of use and publishing policy can be found at https://www.scienceopen.com/.

Preprint statement: This article is a preprint and has not been peer-reviewed, under consideration and submitted to ScienceOpen Preprints for open peer review.

DOI: 10.14293/S2199-1006.1.SOR-.PP2HOHN.v1

Preprint first posted online: 11 December 2021

Keywords: COVID-19, Internet, Older adults, Nursing homes, Long-term-care, Corona, Switzerland 


\title{
Growth in Internet Usage During the COVID-19 Pandemic among Older Adults—Findings from Switzerland
}

Alexander Seifert (University of Zurich)[11.12.2021]

\begin{abstract}
Digital solutions (e.g., videocast) to stay in contact have been more prevalently used during the pandemic; however, have older adults - who are often not familiar with modern technologies - used the internet more often during the pandemic? This brief note presents the first findings about the question of growth in internet use among older adults based on two representative surveys in Switzerland. The results showed that among the community-dwelling older adults, $45.7 \%$ had increased their internet usage, but only $19.5 \%$ among the residents of long-term care facilities had. Social workers should remain aware of the digital divide when working with older adults.
\end{abstract}

Keywords: COVID-19, internet, older adults, Switzerland

The ongoing COVID-19 pandemic affects us all. Every day, we read the latest figures of those suffering from COVID-19 and publicly discuss which measures will best prevent an uncontrollable increase in cases. Since the beginning of the pandemic, a wide range of national and international measures have indicated that people aged 65 and over constitute a particularly vulnerable group (Amadasun, 2020; Shahid et al., 2020). During this pandemic, older adults have had to manage everyday tasks while social distancing and have perhaps even become newly dependent on help from others, such as neighbors or friends (Armitage and Nellums, 2020). While these circumstances may also apply to younger people worldwide, older adults in particular have found themselves in a situation where they have become significantly less connected to the world (Seifert, 2021). This is because older adults do not use the internet as often as younger people and therefore cannot compensate for the reduction in real social contact with digital methods of communication (Seifert et al., 2021). Nonparticipation in the 
digital world during times of physical distancing, and thus isolation, may intensify feelings of social exclusion (Seifert et al., 2018, 2021).

However, have older adults who already use the internet done so more often during the pandemic than before the pandemic? This is the research question to be addressed by this brief report. While younger adults have used the Internet more frequently during the pandemic (Mcclain et al., 2021), older people have seen only a slight increase (Gallistl et al., 2021). Nevertheless, there is a lack of robust data in the context of community-dwelling older adults and those living in long-term care facilities (LTCF).

\section{Research Objectives}

The first research question is, "What growth in internet usage has been indicated by older adults?" We hypothesized that older adults are showing only little growth in internet use during the pandemic. Second, previous research has also shown that age, gender, and education predict the use of the internet among older adults (König et al., 2018; Seifert et al., 2017; Seifert and Cotten, 2020). Therefore, our second research question is. "How does age, gender, and education affect the growth in internet use among older adults?" We hypothesized that younger people, males, and people with more education will show a higher growth in internet use during the pandemic than older people, females, and people with less education.

\section{Data and methods}

\section{Data}

An important characteristic of this secondary data analysis is that it relies on two large surveys performed in Switzerland and presents data from community-dwelling older adults and residents of LTCF. 
In the first survey — a survey about education in old age—1004 people aged 60 years and older filled out an online questionnaire (people without an internet connection or who wanted to complete a written questionnaire could participate with a paper-pencil questionnaire via mail) in November 2020 (Seifert, Perrig-Chiello \& Martin, 2021). A random sample of the permanent resident population aged 60 years and older in Switzerland was selected from the AZ-Direct database (based on the public phonebook). The response rate to the survey was $17.1 \%$. For the analyses, we only included the persons who used the internet; therefore, our sample for analyses included 913 participants. The age of the respondents ranged from $60-95$ years, with a mean age of 71.2 years; $41.4 \%$ were women.

In the second survey — a survey about living conditions in long-term care -876 residents of LTCFs, called Alterszentren in Zurich (which are municipal retirement homes common in Switzerland), were surveyed (Seifert, 2021). Typically, older adults enter these LTCFs having less nursing care at the time of entry, but they can opt to have nursing care until the end of their lives. In May 2020, all 1,614 residents at 22 Alterszentren were approached to request that they complete an eight-page standardized questionnaire with closed-ended questions, predominantly to evaluate their satisfaction with living in the retirement home. Of the 1,614 people who were asked to respond, 876 returned the questionnaire (54.3\% response rate). For this study's sample, only persons who used the internet were included to have a sample comparable to that in the first survey. In the end, the data on 220 people were analyzed for this study. The age of the selected respondents ranged from 56-97 years, with a mean age of 84.1 years; 60.6\% were women. 
All participants approved their participation within the two surveys, and the surveys were conducted under the framework of the Ethics Council of the Philosophy Faculty of the University of Zurich.

\section{Measurement}

The dependent variable was the growth in internet use, which was evaluated subjectively. Within the first study, the answer to the evaluation of the following statement was used to determine internet use: "Since the beginning of the COVID-19 pandemic, I have used the internet more frequently than before." Respondents provided answers using a 5-item Likert scale with scores ranging from 1 ("I totally disagree") to 5 ("I totally agree"). The mean of the scale was 3.25 (standard deviation $[\mathrm{SD}]=1.26$ ). Within the second study, the answers to the following question were used: "Since the pandemic, have you used the internet for social contact more often than before?" The response options were "Yes, more often," "about the same amount," and "no, rather less."

A set of predictor variables established in previous research was considered to explain growth in internet usage. The following sociodemographic variables were included: gender $(0=$ male; $1=$ female $)$, age $($ in years $)$, and education $(0=$ compulsory education and secondary level; 1 = tertiary level).

\section{Statistical Analysis}

SPSS version 24 (IBM Statistics, Amos, NY, USA) was used for statistical analysis. Besides descriptive analyses, multivariate linear regressions for Study 1 and a binary logistic regression for Study 2 were calculated to analyze the predictors of growth in internet use. 


\section{Results}

In Study 1, 27.0\% "rather agreed," and an additional 18.7\% "totally agreed" regarding the statement "Since the beginning of the COVID-19 pandemic, I have used the internet more frequently than before" (Table 1). However, $26.8 \%$ also answered "only partly," and $27.5 \%$ disagreed with the statement, showing only a partial growth in internet use for around $45.7 \%$ of the sample. Female participants reported a higher growth in internet use than male participants (Table 1).

In Study 2, only $19.5 \%$ of internet users in LTCFs reported that they used the internet more often, $56.4 \%$ reported that they used the internet the same amount, and $24.1 \%$ reported that they used the internet rather less compared to the beginning of the pandemic (Table 1). Additionally, in Study 2, female participants reported higher growth in internet use than male participants (Table 1).

\section{[around here Table 1]}

In addition to the descriptive analyses, multivariate analyses were performed to look at the multivariate influence of the predictors (age, gender, and education). First, linear regression analyses were performed with the data from Study 1. The dependent variable was the scale of growth in internet use, while the independent variables included standard demographic factors (age, gender, and education). At the multivariate level, gender was the only significant predictor of growth in internet usage. Female participants used the internet more often during the pandemic than before in comparison to male participants. Second, binary logistic regression analyses were performed with the data from Study 2. The dependent variable was the growth in internet use $(1=$ more often use vs. $0=$ same amount or less often). At the multivariate level, no significant predictors of growth in internet usage were found. 


\section{Discussion}

This study's findings are based on two fairly large samples of older adults living in private households and LTCFs. Our aim was to add to an emerging field that addresses internet use by older adults in such settings and their digital inclusion during a pandemic.

A major finding of our study is that only a modest number of people used the internet more often during the pandemic compared to before the pandemic. Within Study 1 -among the community-dwelling older adults—we found $45.7 \%$ had increased their internet usage, and within Study 2 - among the residents of long-term care institutions - the figure was 19.5\%. Comparing Studies 1 and 2, we found a higher range of growth in internet usage among community-dwelling older adults than among LTCF residents. This shows that internet use among older adults is depended on the living context. Here, residents of LTCFs showed, in general, lower internet or modern technology use and also usable internet service within their institutions than community-dwelling older adults (Schlomann et al., 2020).

Study 1 additionally showed — within the multivariate analyses — that female participants more often reported a growth in internet usage compared to male participants. This demonstrates that maybe older females use the internet more often for social contact, one facet of life that has been limited due to the pandemic, as other studies have shown (Seifert and Hassler, 2021).

\section{Limitations}

Any generalizations of our findings would be limited because of the study's specific regional focus on Switzerland. Although Swiss retirement homes represent a long-term institutionalized care setting, any comparability with settings such as dementia-specific nursing homes or intensive care facilities would be limited. Important 
sociodemographic factors (e.g., income, marital status, and job biography) and psychological factors (e.g., technology biography, self-efficacy, technology anxiety, and personality) were unavailable. Participants were not asked about their purposes in using the internet. Therefore, further research should include information on the use of the internet, examining whether older adults use the internet predominantly for social interactions (such as to converse with their children/grandchildren) or informationseeking, entertainment, or other purposes during the pandemic. The study's crosssectional design did not allow for causal inferences, meaning that longitudinal data on internet use, and the predictors/outcomes of that use, will be necessary in future studies to explicate these relationships and how they may change over time (even after the pandemic).

\section{Conclusion}

The present study is the first to infer that internet usage has not grown much among older adults, especially those living in long-term care institutions, in Switzerland during the COVID-19 pandemic. In conclusion, internet usage may indeed play a causal role in social inclusion in our internet-dominated everyday lives; policies toward improving internet use skills in older adults may also help to reduce the ever-expanding digital divide. Knowing that older adults use the internet less often should remind us of their digital exclusion, meaning when all official information and communication are shared online, we exclude those people who are not "online." 


\section{References}

Amadasun S (2020) Social work and COVID-19 pandemic: An action call. International Social Work: 002087282095935. DOI: 10.1177/0020872820959357.

Armitage R and Nellums LB (2020) COVID-19 and the consequences of isolating the elderly. The Lancet Public Health 5(5): e256. DOI: 10.1016/S24682667(20)30061-X.

Gallistl V, Seifert A and Kolland F (2021) COVID-19 as a "Digital Push?" Research Experiences From Long-Term Care and Recommendations for the Postpandemic Era. Frontiers in Public Health 9. Frontiers. DOI:

10.3389/fpubh.2021.660064.

König R, Seifert A and Doh M (2018) Internet use among older Europeans: an analysis based on SHARE data. Universal Access in the Information Society 17(3): 621-633. DOI: 10.1007/s10209-018-0609-5.

Mcclain C, Vogels E a, Perrin R, et al. (2021) The Internet and the Pandemic. Pew Research Center. Available at: file://Users/alexanderseifert/Downloads/PI_2021.09.01_COVID-19-andTech_FINAL.pdf (accessed 12 November 2021).

Schlomann A, Seifert A, Zank S, et al. (2020) Assistive Technology and Mobile ICT Usage Among Oldest-Old Cohorts: Comparison of the Oldest-Old in Private Homes and in Long-Term Care Facilities. Research on Aging 42(5-6): 163-173. DOI: $10.1177 / 0164027520911286$.

Seifert A (2021) Older adults during the COVID-19 pandemic - Forgotten and stigmatized? International Social Work: 1-4. DOI: 10.1177/0020872820969779.

Seifert A and Cotten SR (2020) In Care and Digitally Savvy? Modern ICT use in Long-Term Care Institutions. Educational Gerontology 46(8): 473-485. DOI: https://doi.org/10.1080/03601277.2020.1776911.

Seifert A and Hassler B (2021) Der Einfluss der COVID-19-Pandemie auf soziale Kontakte bei älteren in der Schweiz wohnhaften Personen. NOVAcura 52(3): $47-50$. 
Seifert, A., Perrig-Chiello, P., \& Martin, M. (2021). Bildungs- und Lernbedürfnisse im Alter-Bericht zur nationalen Befragungsstudie in der Schweiz. Zürich: U3/VSV/SAGW.

Seifert, A. (2021). Bewohner*innen-Befragung ASZ 2021. Zürich: Zentrum für Gerontologie.

Seifert A, Doh M and Wahl H-W (2017) They also do it: Internet use by older adults living in residential care facilities. Educational Gerontology 43(9): 451-461. DOI: $10.1080 / 03601277.2017 .1326224$.

Seifert A, Hofer M and Rössel J (2018) Older adults' perceived sense of social exclusion from the digital world. Educational Gerontology 44(12): 775-785. DOI: $10.1080 / 03601277.2019 .1574415$.

Seifert A, Cotten SR and Xie B (2021) A Double Burden of Exclusion? Digital and Social Exclusion of Older Adults in Times of COVID-19. The Journals of Gerontology: Series B Carr D (ed.) 76(3): e99-e103. DOI: 10.1093/geronb/gbaa098.

Shahid Z, Kalayanamitra R, McClafferty B, et al. (2020) COVID-19 and Older Adults: What We Know. Journal of the American Geriatrics Society 68(5): 926-929. DOI: $10.1111 /$ jgs.16472. 
Table 1: Growth in internet use during the COVID-19 pandemic

\section{Study 1}

\begin{tabular}{lccc}
\hline & Statement: & "Since the beginning of the COVID-19 pandemic, I have used the internet \\
more frequently than before." & Only male \\
\hline Scale & All $(n=913)$ & Only female & $13.5 \%$ \\
\hline I totally disagree & $12.0 \%$ & $9.5 \%$ & $18.3 \%$ \\
I rather disagree & $15.5 \%$ & $11.7 \%$ & $28.8 \%$ \\
Partly agree & $26.8 \%$ & $23.8 \%$ & $24.4 \%$ \\
I rather agree & $27.0 \%$ & $30.6 \%$ & $14.9 \%$ \\
I totally agree & $18.7 \%$ & $24.4 \%$ & \\
\hline
\end{tabular}

\section{Study 2}

\begin{tabular}{lccc}
\hline Statement: & "Since the pandemic, have you used the Internet for social contact more often \\
than before?" & Only female & Only male \\
\hline Yes, more often & All $(n=220)$ & $22.0 \%$ & $16.3 \%$ \\
About the same amount & $19.5 \%$ & $50.0 \%$ & $65.1 \%$ \\
No, rather less & $56.4 \%$ & $28.0 \%$ & $18.6 \%$ \\
\hline
\end{tabular}


Table 2: Regression analysis for the predictors of growth in internet use

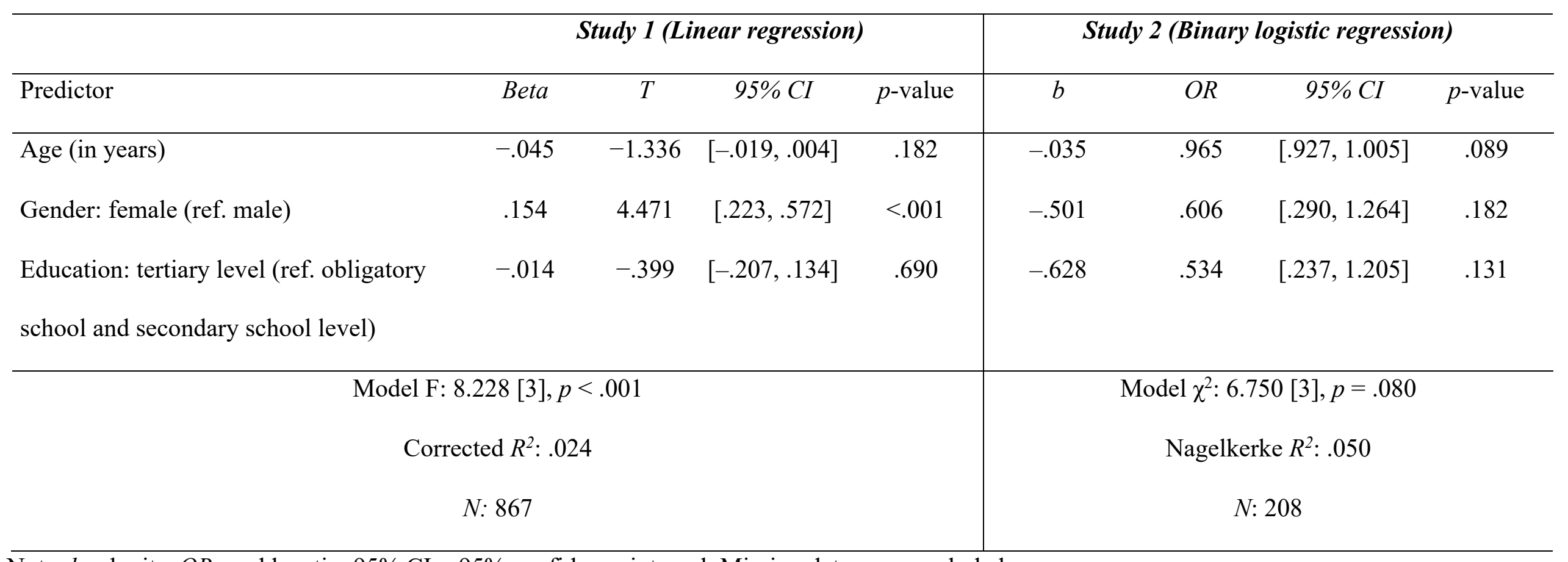

Note: $b=$ logits. $O R=$ odds ratio. $95 \% \mathrm{CI}=95 \%$ confidence interval. Missing data were excluded. 